\title{
Investigating student understanding of cross products in a mathematical and two electromagnetism contexts
}

\author{
T. Deprez, ${ }^{*}$ S. E. Gijsen, ${ }^{\dagger}$ and J. Deprez \\ Department of Mathematics, KU Leuven, Celestijnenlaan 200B, 3001 Leuven, Belgium \\ M. De Cock \\ Department of Physics and Astronomy, KU Leuven, Celestijnenlaan 200D, 3001 Leuven, Belgium
}

(Received 12 September 2018; published 24 September 2019)

\begin{abstract}
We investigated the influence of context on students' understanding of cross products of vectors using three isomorphic multiple-choice tests asking for the direction of a cross product in different geometrical settings. One version of the test involved the Lorentz force, the second version involved the torque on an electric dipole, and the third version was without physics context. We administered the tests to 216 first-year pre-med students at a Belgian (Flemish) university. We found that students perform significantly better in the context of the Lorentz force. Students more often chose the incorrect alternative corresponding to the vector sum in the test versions involving an electric dipole or without physics context when the vectors are not orthogonal. For orthogonal vectors, a sign error-i.e., inverting the direction of the resulting vector-was the most common mistake in both tests with physics context, while without physics context selecting the alternative corresponding to the sum remained the most common mistake. Prior familiarity with a right-hand rule in a specific context seems to be able to explain improved scores in the test version concerning the Lorentz force. Instructors and curriculum developers can benefit from adopting an integrated approach in which the mathematical aspects of the cross product are treated together with multiple examples in physics, allowing students to transition from using specific rules to determine a cross product, to a more integrated understanding of it.
\end{abstract}

DOI: 10.1103/PhysRevPhysEducRes.15.020132

\section{INTRODUCTION}

Previous research has shown that many incoming students at the university level lack basic knowledge of vectors that they need to tackle their physics subjects successfully [1]. In recent years, a number of studies explored students' difficulties with a large variety of vector concepts in detail [2-17].

Relevant to our research are those studies that investigate the effect of a physics context both on performance and on problem-solving processes of the students, when confronted with a problem requiring manipulations of vectors. Regarding vector addition and subtraction, literature reports mixed results. Nyugen and Meltzer [2] found that students spontaneously use the concept of forces on an object when solving problems involving vector addition. Van Deventer and Wittman [3] and Barniol and Zavala [4] discovered that adding a displacement or velocity context

\footnotetext{
*tobe.deprez@kuleuven.be

†stief.gijsen@kuleuven.be
}

Published by the American Physical Society under the terms of the Creative Commons Attribution 4.0 International license. Further distribution of this work must maintain attribution to the author(s) and the published article's title, journal citation, and DOI. improves students' performance on vector addition questions. On the other hand, Shaffer and McDermott [5] reported worse results in vector subtraction when questions were posed in a kinematics context. Flores and Kanim [6] furthermore add that emphasis on vector addition and subtraction in a kinematics course could only partially mitigate students' problems regarding the vector nature of velocity and acceleration. So, the nature of the physics context seems to matter. Emigh et al. [7], however, found that the types of incorrect reasoning students made were roughly similar for different contexts.

At the undergraduate physics level, students learn several physical concepts that give meaning to the cross product of two vectors (torque and Lorentz force among others). Cross products without a physics context is featured in three questions on the "Test of understanding of vectors" by Barniol and Zavala [8]. The authors concluded that students find both calculating a cross product algebraically, as well as finding its direction graphically, difficult. This conclusion was supported by Susac et al. [9] who validated the test of understanding of vectors on a different group, using the Rasch model. In a graphical setting, the most common error when determining the direction of the cross product was a "sign error," i.e., inverting the direction of the resulting vector. Students' performance on determining the direction of the cross product in the context of the 
Lorentz force on a charged particle in a magnetic field has been studied by Scaife and Heckler [10,11]. They also report the occurrence of nonsystematic sign errors. Van Deventer [12] compared students' performance on a question asking for the direction of the cross product between a physics (torque) and math context, and found that students did better when a physics context was given. Kustusch [13] analyzed the effect of numerous problem features, including the presence of a physics context, on students' performance on questions involving determining the direction of the cross product. Among many predictors, it seemed that the role of context was not significant. However, due to the small number and nature of the presented tasks (Van Deventer) or the small number of test subjects (Kustusch), no hard verdict can be given on the role of context on the results.

We report on a large sample study $(N=216)$ on students' performance on a test of seven multiple-choice questions that involve determining the direction of the cross product of two vectors given in graphical representation. There were three versions of the test, each in a different context: one in a purely mathematical setting, one in the context of the Lorentz force on a charge particle in a uniform magnetic field, and one in the context of the torque on an electric dipole in an electric field. Each student solved only one version of the test. We compared students' answers on the three different versions. Our objectives were as follows: (i) to compare students' performance on multiple-choice problems that involve determining the direction of the cross product of two vectors in graphical representation with and without a physics context, (ii) to investigate whether the nature of the physics context influences the performance of students on such problems, (iii) to detect common mistakes students make when solving such problems, and (iv) to investigate whether these mistakes are affected by the context the questions are posed in.

In Sec. II we describe the design and administration of the test. Section III discusses (a) the composition of the test group, (b) the overall performance on the test, and the effects of both context and the background of the students on performance, and (c) how the students responded to each question separately and whether common errors could be identified. Section IV summarizes our findings and compares them to earlier results found in the literature.

\section{METHODOLOGY}

\section{A. Question design}

We designed three isomorphic multiple choice tests, each consisting of seven questions about the direction of the cross product. Because of the limited test time available to us, we restricted ourselves to questions involving the arrow representation as this representation is both ubiquitous in physics courses, and causes a lot of difficulties for students (see, e.g., Heckler and Scaife [16]). The first six questions are "forward problems" in which the students are given two vectors and are asked to select the vector corresponding to the direction of the cross product. The seventh question is a "backward problem" in which the second factor and the cross product are given, and the first factor is sought. The questions represent different geometrical situations: we included five questions where both given vectors lie in the plane of the paper, one for each type of angle (acute, right, obtuse, bigger than $180^{\circ}$ and $0^{\circ}$ ), as well as two questions where one of the given vectors is perpendicular to the plane of the paper.

The three tests differ in context: one test consists of purely mathematical problems, two have a physics context. Both physics contexts come from electricity and magnetism: the Lorentz force on a charged particle moving in a magnetic field and the torque on an electric dipole in an electric field. The resulting three versions of the test are referred to below as math, Lorentz, and dipole, respectively. At the beginning of each test, the formula the students have to use is recalled (e.g., $\vec{F}=q \vec{v} \times B$ for the Lorentz version). Note that in the two tests with physics context, we chose to draw the field lines instead of the field vectors, following convention. Neither did we use the pole representation (i.e., the representation where the north and south pole of a magnet are indicated) as, for example, Scaife and Heckler [10,11] did. Sticking to one conventional representation that students know well allows us to focus on our research objectives, i.e., to assess the role of context on student performance. In the Lorentz test we included the additional aspect that the charge of the moving particle can be positive or negative. An example of a test question for the three different test versions can be found in Fig. 1. Details for each question about the orientation of and the angle between the vectors, as well as sign of the charge in the Lorentz version and the provided alternatives can be found in Table I. The three tests are included in the Supplemental Material [18] of this paper.

For each question we provided five alternatives. The distractors reflect what we anticipated would be common errors. Where applicable we included (i) a vector pointing opposite to the cross product (as previous research revealed a prevalence of sign errors when students determine the direction of a cross product $[8,10,11,13]$ ), (ii) a vector pointing between the given vectors (as in a sum; this distractor also featured in Barniol and Zavala [8]), (iii) a vector pointing opposite to this vector (for symmetry reasons), and (iv) the zero vector. Exceptions occur when the given vectors are parallel and the cross product is the zero vector, or when one of the given vectors is perpendicular to the plane of the paper. In the former case, we included alternatives where the resulting vector points into and out of the plane of the paper (as that would be the cross product if the given vectors were not parallel) and alternatives where the resulting vector points in the same direction and opposite to both given vectors. In the case 
The velocity vector $\vec{v}$ of an electron (with negative charge) makes an angle of $60^{\circ}$ with the magnetic field in which it moves.

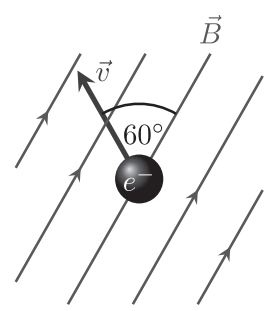

Which figure or proposition represents the correct direction of the force $\vec{F}$ on the electron?

A.

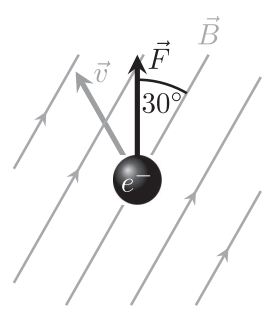

B.

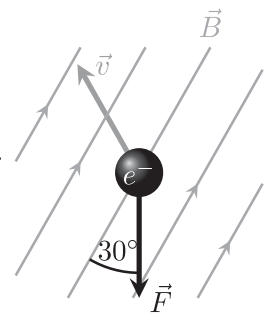

C.

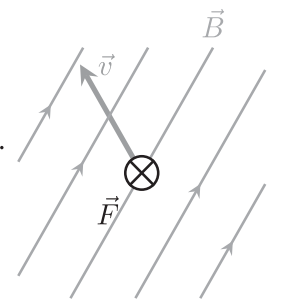

D.

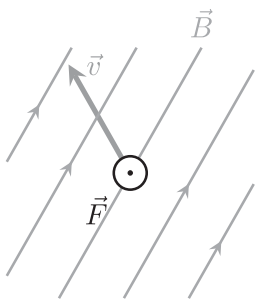

E. $\vec{F}$ is the zero vector

The electric dipole moment $\vec{p}$ makes an angle of $60^{\circ}$ with the electric field in which it lies.

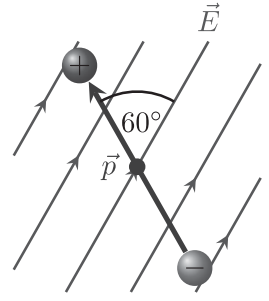

Which figure or proposition represents the correct direction of the torque $\vec{\tau}$ on the electric dipole?

A.

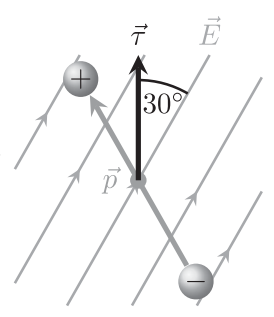

B.

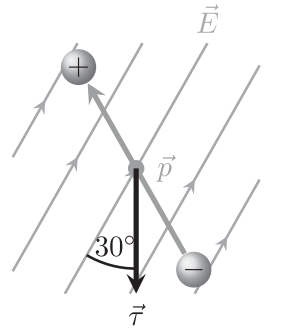

C.

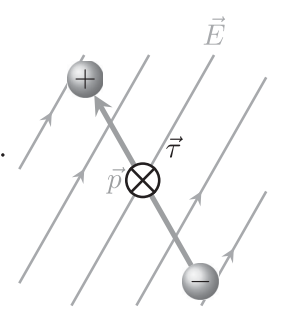

D.

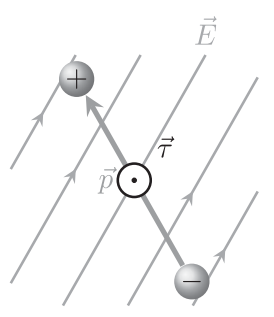

E. $\vec{\tau}$ is the zero vector

The vector $\vec{A}$ makes an angle of $60^{\circ}$ with the vector $\vec{B}$.

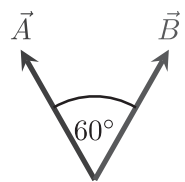

Which figure or proposition represents the correct direction of the cross product $\vec{C}=\vec{A} \times \vec{B}$ ?

A.

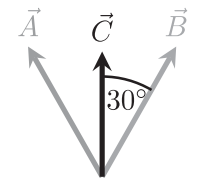

B.

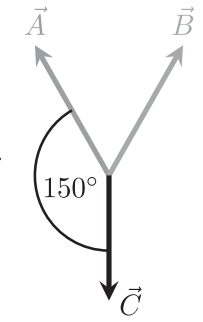

C.

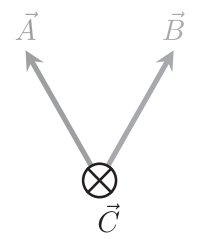

D.

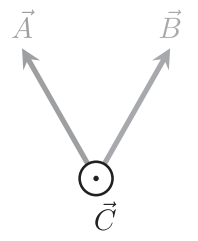

E. $\vec{C}$ is the zero vector

FIG. 1. Example of a question used in the test. Above the Lorentz version, in the middle the dipole version and below the math version. On the test, the five answer alternatives were ordered randomly for each question. 
TABLE I. Overview of the orientation of, and the angle between the vectors in seven questions on the test, as well as the sign of the charge in the Lorentz version and the alternatives the students could chose from. The correct alternatives are indicated in bold, or in italics for the Lorentz version if it is different from the other test versions.

\begin{tabular}{|c|c|c|c|c|c|}
\hline \multirow[b]{2}{*}{ No. } & \multicolumn{2}{|c|}{ Orientation } & \multirow[b]{2}{*}{ Angle } & \multirow[b]{2}{*}{ Charge in Lorentz } & \multirow[b]{2}{*}{ Alternatives } \\
\hline & First & Second & & & \\
\hline 1 & north & east & $90^{\circ}$ & positive & $\begin{array}{l}\text { into paper, out of paper, in between, opposite to } \\
\text { in between, zero vector }\end{array}$ \\
\hline 2 & north west & north east & $60^{\circ}$ & negative & $\begin{array}{l}\text { into paper, out of paper, in between, opposite to } \\
\text { in between, zero vector }\end{array}$ \\
\hline 3 & south west & north east & $204^{\circ}$ & positive & $\begin{array}{l}\text { out of paper, into paper, in between, opposite to } \\
\text { in between, zero vector }\end{array}$ \\
\hline 4 & north & north & $0^{\circ}$ & positive & $\begin{array}{l}\text { zero vector, into paper, out of paper, same as factors, } \\
\text { opposite to factors }\end{array}$ \\
\hline 5 & south east & north & $120^{\circ}$ & negative & $\begin{array}{l}\text { out of paper, into paper, in between, opposite to } \\
\text { in between, zero vector }\end{array}$ \\
\hline 6 & north east & out of paper & $90^{\circ}$ & negative & $\begin{array}{l}\text { south east, north west, into paper, out of paper, } \\
\quad \text { zero vector }\end{array}$ \\
\hline \multirow[t]{2}{*}{7} & asked & into paper & $90^{\circ}$ & positive & $\begin{array}{l}\text { south west, north east, into paper, out of paper, } \\
\text { zero vector }\end{array}$ \\
\hline & product ve & south east & & & \\
\hline
\end{tabular}

where one of the given vectors is perpendicular to the plane of the paper, we chose to give alternatives where the resulting vector is parallel to this vector (i.e., pointing into or out of the paper). The alternatives were ordered randomly (with different order for each test version).

In a small pilot study, we administered the tests to 21 second-year mathematics majors, asking them to solve the questions and to provide (written) feedback. We mainly did this in order to ensure all problems were formulated clearly: the small number of test subjects meant further analysis would be speculative. We did not interview any of the students. Other than underlining the charge of the particle in the Lorentz version, no significant changes were made after this pilot study.

\section{B. Administration}

We administered the test to a group of 216 first year premed students at a Belgian (Flemish) university in the midst of their second semester, during a calculus-based introductory course on electricity and magnetism. In their first semester the students had a course covering mechanics (including rotational motion), oscillations, and waves. At the moment of the test, the Lorentz force and the electric dipole moment had already been discussed in the lectures.

It is worthwhile to note that students in Flanders first encounter the Lorentz force, as well as a right-hand rule specific for this context, in secondary school (i.e., the form of education before entering university) without explicitly referring to the concept of cross product. This right-hand rule is a special case of the typical right-hand rule for cross products where the thumb, index finger, and middle finger represent the vectors in the cross product (in this case the velocity vector, the magnetic field vector, and the resulting force vector). The cross product itself is only taught at university, for the students in question in the first-term mechanics course. Also the electric dipole is not taught in secondary school.

To avoid that questions from one context influenced the answers students chose on similar questions in other contexts, we chose to let each student solve only one of the three tests. The assignment of the students to the test versions was done randomly, allowing us to assume the three test groups are statistically similar. The students took the test anonymously, though we asked them to provide some background information: gender, number of math classes per week in the last years of secondary school and whether they passed the first-term physics course. The last two variables are referred to as weekly hours math and passed mechanics course below. Note that it is possible for students to take this particular second-term physics course while not having passed the first-term course yet.

We administered the test during the final 20 min of one of the lectures of the electromagnetism course. All students have the same lecturer and follow lectures in a large auditorium. (They have additional exercise classes in smaller groups.) All students who were present during the lecture took part. The test did not count for the grades of the students. The students filled out the test individually. They did not consult any notes while taking the test. We asked the students to leave no questions blank, but instead to select the answer that seemed most probable to them in case they were not sure. Almost all students were able to complete the test within $20 \mathrm{~min}$.

\section{RESULTS}

In this section we discuss the students' answers on the test. We start by summarizing the composition of our test group, based on the background information we asked for on the 
TABLE II. $p$ values for chi-squared tests on each pair of the variables test version, gender, weekly hours math, and passed mechanics course. All pairs can be assumed to be independent, except weekly hours math and passed mechanics course.

\begin{tabular}{lccc}
\hline \hline & & $\begin{array}{c}\text { Weekly } \\
\text { hours math }\end{array}$ & $\begin{array}{c}\text { Passed mechanics } \\
\text { course }\end{array}$ \\
\hline Test version & 0.3752 & 0.8617 & 0.9658 \\
Gender & N/A & 0.2411 & 0.2323 \\
Weekly hours math & & N/A & 0.00727 \\
\hline \hline
\end{tabular}

test. Next, we discuss students' overall performance on the test. Finally, we discuss students' answers on each question.

\section{A. Composition of test group}

Of the test group of 216 students, twelve did not provide complete answers to all questions or did not provide all background information. We did not include the answers of these students in the results below.

The test group consisted of 166 women (81\%) and 38 men (19\%). 157 students $(77 \%)$ passed the first-term mechanics course. In the last years of secondary school, 41 students $(20 \%)$ were in a program with less than $6 \mathrm{~h}$ of mathematics per week, $123(60 \%)$ in a program with exactly six hours and $40(20 \%)$ in a program with more than $6 \mathrm{~h}$. It is worthwhile to note that in Flanders a program with $6 \mathrm{~h}$ of mathematics per week is very common for people wanting to follow a scientific program at university level. 68 students (33\%) filled in the Lorentz version, 65 (32\%) the dipole version, and $71(35 \%)$ the math version.

To check whether the variables "test version," "gender," "weekly hours math," and "passed mechanics course" are independent, we used chi-squared tests. The results of these tests can be found in Table II. With threshold $p=0.05$, the variable test version can be assumed to be independent of the three other variables. This confirms that the test was distributed randomly. Furthermore, gender can be assumed to be independent of weekly hours math and passed mechanics course. The variables weekly hours math and passed mechanics course cannot be assumed to be independent.

\section{B. Overall performance on tests \\ 1. Test scores in different contexts}

Scores of the students on the different test versions can be found in Fig. 2.

The best scores were obtained in the Lorentz version. The most frequent score for this version was 3 (24 students, $35 \%$ ), and 46 students (68\%) obtained a score of 3 or higher. Students scored the worst in the dipole version: 1 was the most frequent score (22 students, 34\%), and only 25 students (38\%) scored 3 or higher. The scores on the math version lie in between the other two. Again the most frequent score was 1 (16 students, 23\%), and 31 students $(44 \%)$ scored 3 or higher.

Figure 2 illustrates that the distribution of scores on neither of the test versions can be approximated well by a normal distribution. Therefore we used the nonparametric Kruskal-Wallis test $[19,20]$ to compare the three groups. The test revealed that the scores on the different test versions were significantly different $(p=0.007)$. A Wilcoxon rank-sum test [20] yielded that students who took the Lorentz version scored significantly better than the students who took the dipole and math versions (respectively, $p=0.0007$ and $p=0.01275$ ). The effect size, measured by Cohen's $d$ statistic [21], was found to be of medium size ( $d=0.5174)$ for the Lorentz versus dipole test scores and small $(d=0.3054)$ for the Lorentz versus math test scores. The difference between the dipole and math versions was not significant $(p=0.5582)$.

\section{Influence of gender, weekly hours math, and passed mechanics course on overall test scores}

Aside from the influence of context, we checked whether the variables gender, weekly hours math, and passed mechanics course had an impact on the test scores.

We found that men scored significantly better than women (Wilcoxon rank-sum test, $p=0.04574$ ), but the effect size is small (Cohen's $d=0.4616$ ). The small sample
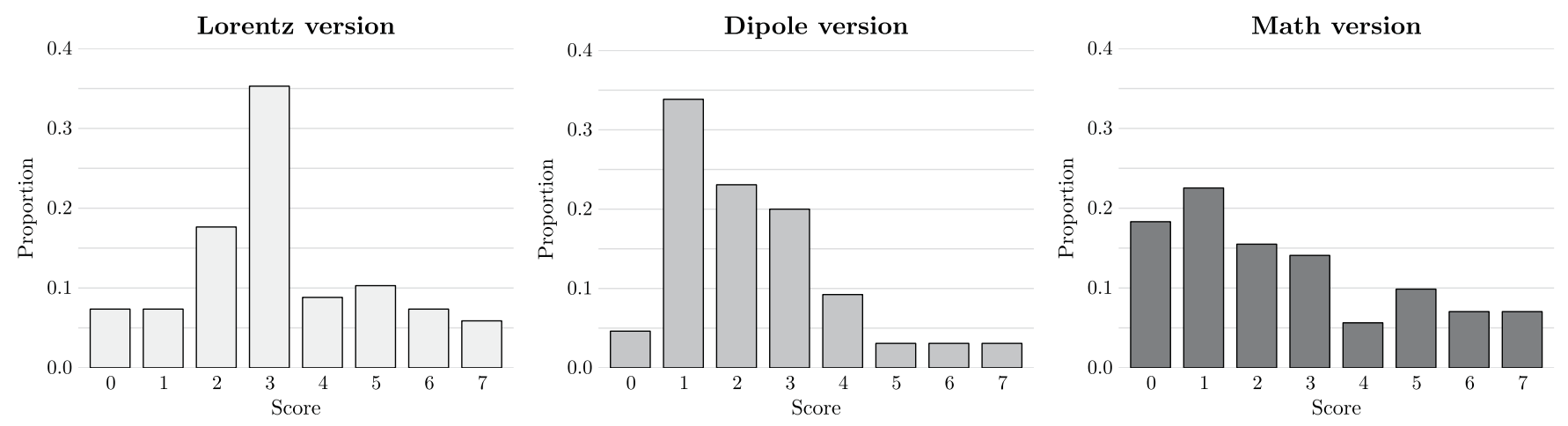

FIG. 2. Students' scores on the Lorentz (left), dipole (middle), and math (right) version. A correct answer on a question counts as one, a wrong answer as zero. 


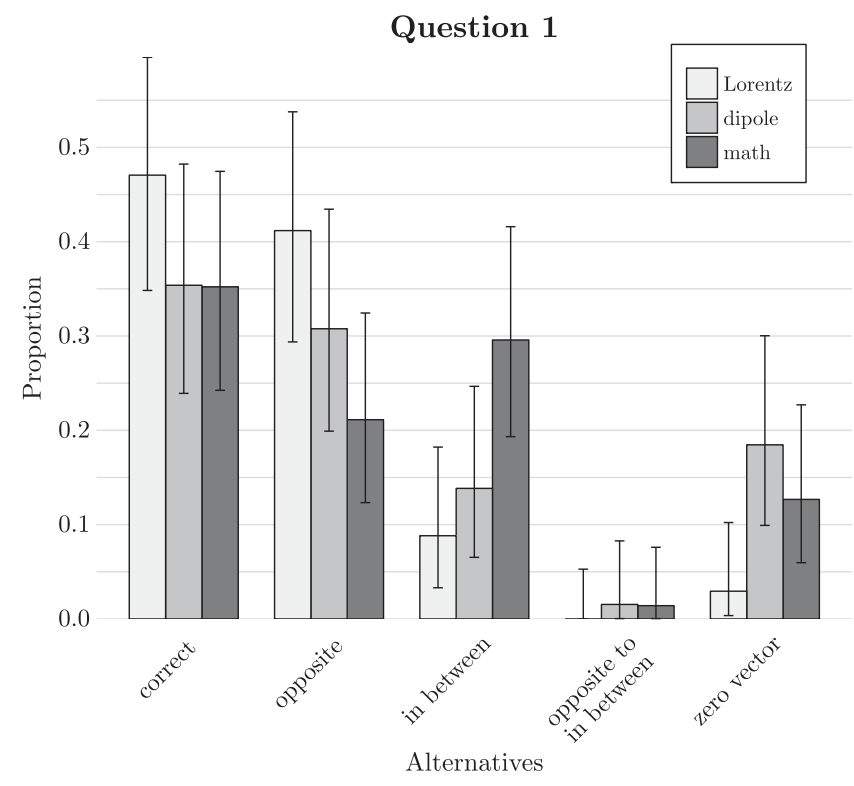

FIG. 3. Students' answers on question 1 about the cross product of two orthogonal vectors. Error bars indicate the $95 \%$ confidence intervals based on the Clopper-Pearson method [22].

size of male students did not allow us to check whether this difference was bigger in certain test versions than in others. Somewhat surprisingly, the influence of the other two variables was not significant. Kruskul-Wallis tests on the students' scores when grouped by weekly hours math, by passed mechanics course and by both variables yielded $p$ values of $0.2055,0.08895$, and 0.09024 , respectively. Since we showed that the variables pertaining to the students' background are independent, it allows us to conclude that differences in scores between genders are not explained by differences in the other two variables.

\section{Students' answers on individual questions}

In order to get more insight in student errors, we investigate students' answers on the individual questions of the test.
Below, we will restrict ourselves to the numbers in our sample and will not make inferences on the population level.

We did not find many students consistently making the same mistake. For instance, there was only one student who consistently selected the alternative corresponding to a sign change.

\section{Cross product of orthogonal vectors in plane of paper}

The first question asked students to indicate the direction of the cross product of two orthogonal vectors in the plane of the paper. Students' answers on this question are shown in Fig. 3. In all versions the correct answer was the most frequent [32 students (47\%) in Lorentz, 23 students (35\%) in dipole and 25 students (35\%) in math]. In both tests with physical context the vector pointing in the opposite direction to the correct vector was chosen almost as frequently as the correct answer [28 students (41\%) in Lorentz, 20 students (31\%) in dipole, and only 15 students $(21 \%)$ in math]. In the math version the second most frequently chosen option was the vector in between the two given vectors, like in the sum (21 students, 30\%). In the other two versions this option was considerably less frequently chosen [6 students $(9 \%)$ in Lorentz and 9 students (14\%) in dipole]. The zero vector was also fairly frequently chosen in the dipole and math version [12 students (19\%) and 9 students (13\%), respectively], but not in the Lorentz version (2 students, $3 \%$ ).

\section{Cross product of nonorthogonal vectors in the plane of the paper}

The answering patterns on the questions where the angles between the two given vectors were acute, obtuse, and bigger than $180^{\circ}$ (questions 2,5 , and 3 , respectively) were very similar to each other, see Fig. 4. The proportion of correct answers on these questions was lower than on the first question. Interestingly, the most frequently chosen answer on the dipole and math versions for these three questions was the vector lying in between the two given vectors (hence

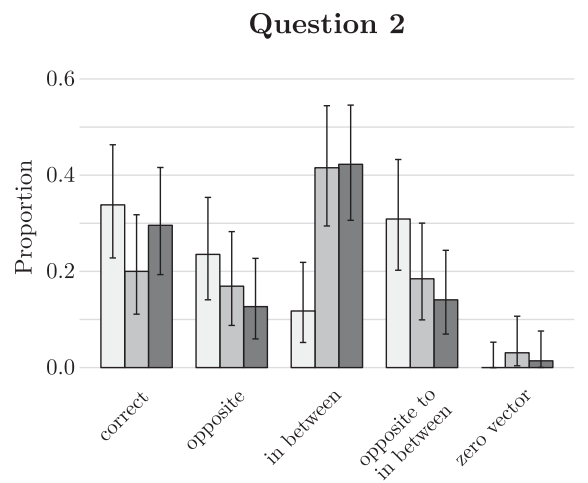

Alternatives

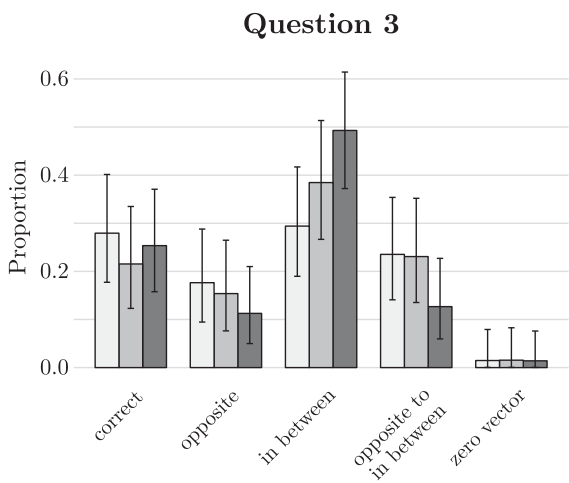

Alternatives

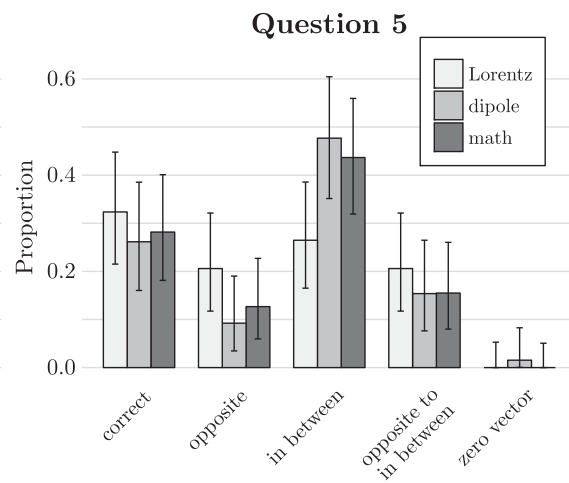

Alternatives

FIG. 4. Students' answers on question 2 (left), 3 (middle), and 5 (right) about the cross product of vectors at an angle of $60^{\circ}, 204^{\circ}$, and $120^{\circ}$, respectively. Error bars indicate the $95 \%$ confidence intervals based on the Clopper-Pearson method. 


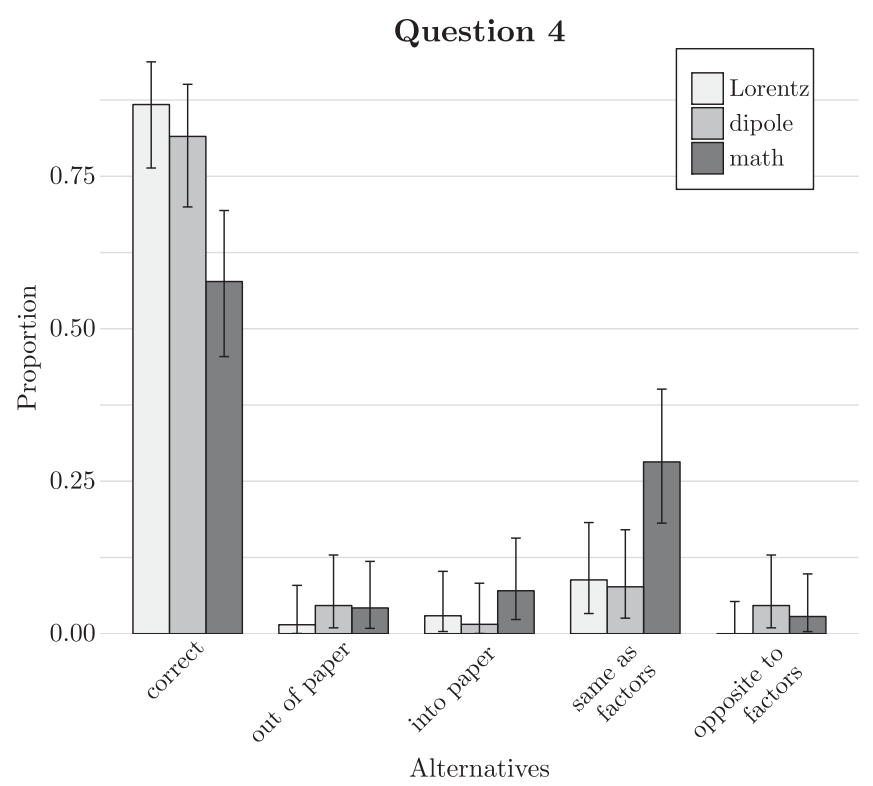

FIG. 5. Students' answers on the question 4 about the cross product of two parallel vectors. Error bars indicate the $95 \%$ confidence intervals based on the Clopper-Pearson method.

neither the correct alternative nor the alternative corresponding to a sign error). On the Lorentz version, the correct answer was more often the most frequent. However, for questions 3 and 5 (obtuse angle and angle bigger than $180^{\circ}$ ) the vector lying in between was almost as frequently chosen as the correct answer. On question 2 (acute angle) this was the case for the alternative pointing opposite to the vector lying in between. On all questions and on all versions the alternative corresponding to the sign change and the alternative pointing opposite to the vector lying in between were also fairly frequently chosen, while the zero vector was almost never chosen. No influence of the sign of the charge could be discerned.

\section{Cross product of parallel vectors}

On question 4, where the two vectors were parallel (see Fig. 5), almost all students in the tests with physics context answered correctly [59 students (87\%) in Lorentz, 53 students $(82 \%)$ in dipole]. In the math version, only 41 students $(58 \%)$ answered correctly. In all versions, the second most frequently chosen alternative was the vector pointing in the same direction as both given vectors [6 students (9\%) in Lorentz, 5 students (8\%) in dipole, and 20 students (28\%) in math].

\section{Cross product with second factor pointing out of the paper}

On question 6, where the second factor pointed out of the plane of the paper (see Fig. 6), most students either selected the alternative corresponding to the correct or the opposite direction. In the Lorentz version 34 students (50\%) chose
Question 6

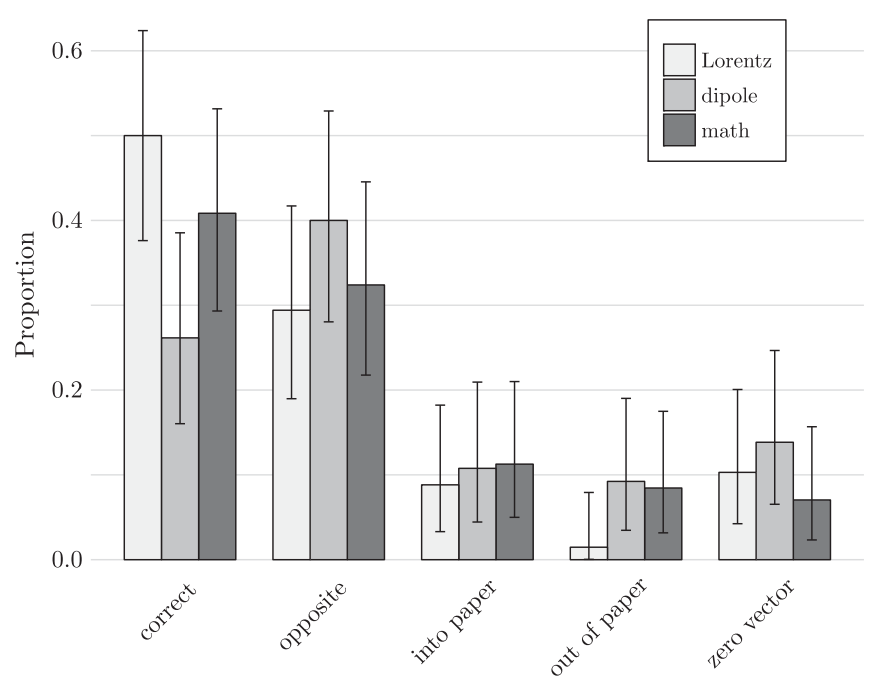

Alternatives

FIG. 6. Students' answers on question 6 about the cross product where the second factor pointed out of the plane of the paper. Error bars indicate the $95 \%$ confidence intervals based on the Clopper-Pearson method.

the correct alternative, and 20 students (29\%) chose the alternative pointing in the opposite direction. In the math version, these were respectively 29 students $(41 \%)$ and 23 students (32\%). Remarkably, in the dipole version more students chose the vector pointing in the opposite direction (26 students, 40\%) than in the correct direction (17 students, 26\%).

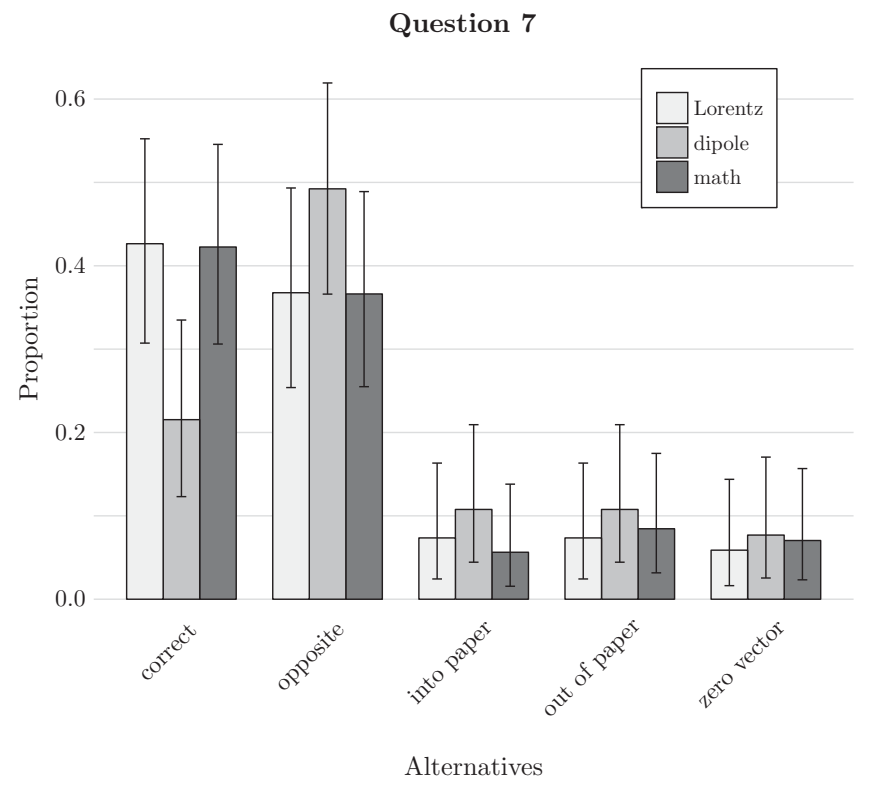

FIG. 7. Students' answers on question 7 where the direction of first factor of the cross product was sought. Error bars indicate the 95\% confidence intervals based on the Clopper-Pearson method. 


\section{Determine first factor of cross product}

On the final question (question 7, see Fig. 7 for students' answers), we asked the students in which direction the first factor of a cross product pointed, when the second factor of the product pointed into the plane of the paper, and the second factor lies in the plane of the paper. The students' answers were similar to the previous question, albeit that slightly less students answered correctly in the Lorentz and dipole version. In the Lorentz version 29 students (43\%) chose the correct alternative, and 25 students (37\%) the vector pointing in the opposite direction. In the dipole version these were 14 students (22\%) and 32 students (49\%), respectively, and in the math version 30 students (42\%) and 26 students (37\%), respectively.

\section{DISCUSSION AND CONCLUSIONS}

The scores on all three test versions were low. This suggests that students struggle a lot with applying a correct technique to determine the direction of a cross product, and doing so appropriately and consistently. The result is surprising considering that students took the test in the middle of a course on electromagnetism, in which cross products and the right-hand rule were used extensively.

We found that the scores on the Lorentz version of the test were significantly higher than on the dipole or math version, while the difference between the scores on the dipole and the math version was not significant. Concerning objectives (i) and (ii) in the introduction, this suggests that the students' performance does not so much depend on whether there is a physics context or not, but more on the nature of the physics context. The physics context of moving charges in a magnetic field helped students in solving the problems correctly. Considering that one also has to take into account the charge of the particle for every problem, which could reverse the orientation of the Lorentz force vector, the improved performance on these questions becomes even more relevant. This is in contrast to results by Kustusch [13] who concluded that the role of context is not significant compared to other test features. Our results on the other hand agree surprisingly well with those of Van Deventer [12], who also found a correct response rate of about $30 \%$ and better performance within a dynamics (torque on a pulley) context.

Perhaps this is explained by the fact that Flemish students learn to use a right-hand rule for the first time in the setting of the Lorentz force, without explicitly referring to the concept of cross product, which is introduced only much later at the university level. It might be that the context of the Lorentz force triggers the students to use the special case of the right-hand rule learned in secondary school instead of the general right-hand rule for cross products. The context of the electric dipole does not trigger such a special rule, forcing the students to rely on the general right-hand rule for cross products.
This could explain the better results for the Lorentz version of the test.

One can apply a similar type of reasoning to the research of Van Deventer [12] and Kustusch [13]. Van Deventer [12] found that students responded correctly more often when asked a question about the torque on a pulley in a dynamics context, compared to an isomorphic question without context. The lack of significance of physics context on student performance found by Kustusch [13] can be explained (as suggested in their article) due to the fact that students in their test group are more familiar with cross products in general. Moreover, in this study questions about two different magnetic contexts (magnetic field generated by moving charges and Lorentz force on a charged particle in a magnetic field) were mixed together, possibly obscuring the effect of the Lorentz force context on student performance.

We can conclude, in relation to research objectives (i) and (ii), that the nature of the physics context seems to matter more than whether or not a physics context is provided. In particular, prior familiarity with a right-hand rule in a specific physics context improves students' performance applying the cross product in that context, but also induces context-specific errors. Unfamiliarity with right-hand rules in a specific context leads to the same performance as if there were no context at all. Finally, more experience with cross products in general can mitigate context dependance. A large-scale qualitative study, comparing students' solution strategies for cross product questions in a variety of physics contexts, would be interesting. This might explain further why the presence of some physics contexts improve student performance on cross product questions while others do not, and how experience with cross products in general affects the solution strategies used by students.

The sign of the charge did not measurably affect student performance on the Lorentz version of the test, in contrast to findings of Kustusch [13]. Regarding the questions with nonorthogonal cross products (questions 2, 3, and 5), the students did not make more sign errors in the Lorentz version on questions 2 and 5 (where the particle had negative charge), both compared to question 3 , as well as compared to the isomorphic dipole or math problems of questions 2 and 5. Note that we did not isolate this problem feature on the test, hence no strict conclusions can be drawn. In light of these contrasting findings, again further research would be interesting to isolate the effect of the sign of the charge on student performance.

Taking a more detailed look into students' answers on the different problems yielded additional insights in relation to objectives (iii) and (iv).

The results on question 1 suggest that the physics context helps students to realize that the cross product vector should be perpendicular to the two given vectors. In the math version, the alternative corresponding to the vector 
sum was clearly more popular than in the versions with physical context. Furthermore, the alternative corresponding to the vector sum was the most common mistake in the math version. This agrees well with results from Van Deventer [12], who also found this to be by far the most picked alternative. In the Lorentz and dipole versions, a sign error was the most common mistake made by students. One possible cause of the sign errors could be that students are confused about the meaning of the symbols $\odot$ and $\otimes$ as discovered by Kustusch [13] (even though these symbols are also used in class). It could have helped to recall these symbols on the test. Another explanation could be that students do not take into account the noncommutativity of the cross product, as suggested by Scaife and Heckler [10]. This would also explain the observation we made earlier concerning the nonsystematic nature of the sign errors, an observation that was also clear in previous studies about cross products $[8,10,13]$.

On questions 6 and 7, the orientation of the vectors and the chosen representation did not allow us to provide alternatives corresponding to the sum of the given vectors. Here, a sign error was the most common error. Comparing questions 6 and 7, it is noteworthy that the type of problem (forward or backward, i.e., which vector was sought) did not affect the performance of the students, in sharp contrast to the findings of Kustusch [13]. A possible explanation is that in the particular case of problem 7, calculating the cross product of the two given vectors (hence treating it as a forward problem) also yielded a vector with the correct orientation (but possibly the opposite direction, depending on which vector was chosen as the first vector). In particular, computing the product $\vec{B} \times \vec{C}$ (or analogues in the physics versions) would yield the correct result. Errors made due to noncommutativity issues would be masked by this.

On question 4 (parallel vectors), the physical context helped students to correctly identify that the product must be zero. In the math version many students thought the product would point in the same direction as the given vectors, suggesting confusion with the sum.

For problems in which the two given vectors were neither orthogonal nor parallel (questions 2, 3, and 5), the correct response rate was lower than on the first question. This confirms results by Kustusch [13] who found that "Angle" is a significant indicator of performance in problems without physics context. In general, a lot of students incorrectly indicated the vector corresponding to the sum. This was especially evident in the dipole and math versions. Our results contrast the findings of Barniol and Zavala [8] who found that a sign error was the most common mistake. Two explanations are possible: either the physical awkwardness of applying some versions of the right-hand rule (in particular, the version where thumb, index finger, and middle finger represent the two ordered factors and the cross product, respectively) leads students to inappropriately apply other techniques, or having the vectors at an angle triggers an association with the vector sum (perhaps influenced by problems in Newtonian dynamics involving force diagrams). In the Lorentz version, errors corresponding to the vector sum appeared less often, and sign errors more often.

We can hence complement our findings regarding research objectives (i) and (ii) with the following conclusions of the qualitative research. Lack of prior experience with a right-hand rule in the dipole context not only results in similar student performance as if there would have been no context at all, also the types of errors madein particular, confusion with the vector sum-are very similar in the dipole and math version. The context of the Lorentz force recalls solution strategies resulting in fewer errors concerning addition of the vectors.

It should be noted that a physics context can also lead to context-specific errors as demonstrated by Van Deventer [12]. The dynamics context they provided, prompted the error that some students indicated that the torque $\vec{\tau}=\vec{r} \times \vec{F}$ on the pulley be directed "counterclockwise" to the position vector $\vec{r}$ and applied force $\vec{F}$.

We therefore recommend that instructors or curriculum developers use an integrated approach when teaching cross products, in which a mathematical and physical treatment go hand in hand, and which use prior knowledge of the students where applicable. Such instruction ideally results in students being able to transfer the different concepts surrounding cross products to different contexts, and makes them aware of context-triggered misconceptions that might occur.

A design choice that might have provided visual confusion for the students is the fact that we chose to depict the charged particles in the Lorentz and dipole version for some, but not all answer alternatives (due to the impossibility of drawing them in the chosen representation). In hindsight it would probably have been better not to include them in the answer options.

Because of the test involving only multiple-choice questions, we cannot know for sure what techniques and thought processes students used to arrive at their answers. Future research combining quantitative and qualitative methods could shed more light on common reasonings and misconceptions students have about cross products and right-hand rules.

\section{ACKNOWLEDGMENTS}

The authors would like to thank Jan Fissette and Thomas Goossens for their contribution to this work. This publication was made possible through funding support of the KU Leuven Fund for Fair Open Access. 
[1] R. D. Knight, The vector knowledge of beginning physics students, Phys. Teach. 33, 74 (1995).

[2] N.-L. Nguyen and D. E. Meltzer, Initial understanding of vector concepts among students in introductory physics courses, Am. J. Phys. 71, 630 (2003).

[3] J. Van Deventer and M. C. Wittmann, Comparing student use of mathematical and physical vector representations, AIP Conf. Proc. 951, 208 (2007).

[4] P. Barniol and G. Zavala, Vector addition: Effect of the context and position of the vectors, AIP Conf. Proc. 1289, 73 (2010).

[5] P. S. Shaffer and L. C. McDermott, A research-based approach to improving student understanding of the vector nature of kinematical concepts, Am. J. Phys. 73, 921 (2005).

[6] S. Flores, S. E. Kanim, and C. H. Kautz, Student use of vectors in introductory mechanics, Am. J. Phys. 72, 460 (2004).

[7] P. J. Emigh, G. Passante, and P. S. Shaffer, Student understanding of superposition: Vectors and wave functions (American Association of Physics Teachers, Sacramento, CA, 2016), pp. 112-115, https://doi.org/10.1119/ perc.2016.pr.023.

[8] P. Barniol and G. Zavala, Test of understanding of vectors: A reliable multiple-choice vector concept test, Phys. Rev. ST Phys. Educ. Res. 10, 010121 (2014).

[9] A. Susac, M. Planinic, D. Klemencic, and Z. Milin Sipus, Using the Rasch model to analyze the test of understanding of vectors, Phys. Rev. Phys. Educ. Res. 14, 023101 (2018).

[10] T. M. Scaife and A. F. Heckler, Student understanding of the direction of the magnetic force on a charged particle, Am. J. Phys. 78, 869 (2010).

[11] T. M. Scaife and A.F. Heckler, Interference between electric and magnetic concepts in introductory physics, Phys. Rev. ST Phys. Educ. Res. 7, 010104 (2011).
[12] J. Van Deventer, Comparing student performance on isomorphic math and physics vector representations, master thesis, University of Maine, 2008.

[13] M. B. Kustusch, Assessing the impact of representational and contextual problem features on student use of right-hand rules, Phys. Rev. Phys. Educ. Res. 12, 010102 (2016).

[14] J. M. Hawkins, J. R. Thompson, M. C. Wittmann, E. C. Sayre, and B. W. Frank, Students responses to different representations of a vector addition question, AIP Conf. Proc. 1289, 165 (2010).

[15] P. Barniol and G. Zavala, Force, velocity, and work: The effects of different contexts on students' understanding of vector concepts using isomorphic problems, Phys. Rev. ST Phys. Educ. Res. 10, 020115 (2014).

[16] A. F. Heckler and T. M. Scaife, Adding and subtracting vectors: The problem with the arrow representation, Phys. Rev. ST Phys. Educ. Res. 11, 010101 (2015).

[17] P. Barniol and G. Zavala, Students' difficulties in problems that involve unit-vector notation, Latin Am. J. Phys. Educ. 8, 4403-1 (2014).

[18] See Supplemental Material at http://link.aps.org/ supplemental/10.1103/PhysRevPhysEducRes.15.020132 for the three tests used for this article.

[19] W. H. Kruskal and W. Allen Wallis, Use of ranks in onecriterion variance analysis, J. Am. Stat. Assoc. 47, 583 (1952).

[20] G. W. Corder and D. I. Foreman, Nonparametric Statistics for Non-Statisticians (John Wiley \& Sons, Inc., Hoboken, NJ, 2009).

[21] J. Cohen, Statistical Power Analysis for the Behavioral Sciences (Routledge, London, 2013).

[22] C. J. Clopper and E. S. Pearson, The use of confidence or fiducial limits illustrated in the case of the binomial, Biometrika 26, 404 (1934). 